\title{
Acid-base imbalance and arrhythmias after myocardial infarction
}

\author{
J. Pilcher and R. E. Nagle \\ From Royal Infirmary, Sheffield S6 ${ }_{3} D A$; and Selly Oak Hospital, Birmingham
}

Studies have been made of 142 episodes of myocardial infarction to determine whether there is any relation between admission acid-base status and the incidence of ventricular arrhythmias. No such relation exists in uncomplicated infarction or in infarction complicated by heart failure. Experimental and clinical evidence suggests that for arrhythmias to be provoked by acid-base imbalance this must be gross. Such degrees of imbalance are not attained in myocardial infarction without cardiac arrest or cardiogenic shock. There was an association between cardiogenic shock, agonal arrhythmias, and metabolic acidosis in this study. It seems likely that this association is largely mediated by an inadequate cardiac output provoking both acidosis and arrhythmias, but it is also probable that acidosis, when gross, acts as a secondary agent in arrhythmic production.

Measurement of acid-base balance is of value in assessing probable mortality from myocardial infarction - metabolic acidosis carrying a poor prognosis. It is of no value as a guide to the development of future ventricular arrhythmias.

Metabolic acidosis may occur after myocardial infarction (Neaverson, 1966) and is particularly common in cardiogenic shock (MacKenzie et al., 1964). Experimental evidence suggests that severe acidosis predisposes the heart to arrhythmias (Karis, Harmel, and Hoffman, 1960; Hecht and Hutter, 1964; Gerst, Fleming, and Malm, I966) and prevents successful treatment of ventricular fibrillation (Ledingham and Norman, 1962). Clinical evidence also suggests that such arrhythmias may be caused by alkalosis (Flemma and Young, 1964; Ayres and Grace, 1969).

The purpose of this study has been to correlate acid-base status found after myocardial infarction with the presence or absence of ventricular arrhythmias over the subsequent 24 hours and to determine the effect of severity of infarction upon any such relation. It should then be possible to determine whether acidbase imbalance has any prognostic guide as to the likelihood of development of these arrhythmias.

\section{Patients and methods}

Studies were made on 142 episodes of recent (less than 72 hours) myocardial infarction. There were II 8 men and 24 women whose ages ranged between 33 and 82 (mean age 57 with a standard

Received 19 October 1970. deviation of IO. I years). All had been admitted to a coronary care unit and their live stay within this unit varied between 36 hours and 24 days (mean 5.5 days). A diagnosis of myocardial infarction was confirmed in all patients by a typical history, diagnostic changes in the electrocardiogram, and a transient rise in the relevant enzyme tests.

All patients except 5 were in sinus rhythm without significant ventricular premature beats at the time of onset to the study. Of these 5, 2 were in atrial fibrillation of long standing and 3 were being transvenously paced for complete heart block.

During their stay within the coronary care unit all patients were oscilloscopically monitored with respect to heart rate and rhythm. Nursing staff were trained to recognize and where possible to record any arrhythmia seen on either the individual single channel or central multichannel oscilloscopes.

Arterial samples were taken as soon as possible after admission to the unit and in all cases within 90 hours of the probable onset of infarction. Direct brachial or radial artery punctures were well tolerated without local anaesthetic. At least $5 \mathrm{ml}$ blood were collected into heparinized glass syringes and arterial pressure used in driving back the syringe plunger. These blood samples were estimated personally for $\mathrm{pH}$ and $\mathrm{PCO}_{2}$ using standard Radiometer micro-electrodes at $37^{\circ} \mathrm{C}$. In general, estimation was done immediately after collection and in no case did more than two hours elapse between collection and estimation: such samples were stored on ice pending estimation. Electrode calibration followed the makers' instructions using buffer ampoules for $p \mathrm{H}$ and saturated reference 
gases (Haldane) for $\mathrm{PCO}_{2}$. All samples were estimated in duplicate. These duplicate readings were accepted if they agreed to within $0.0 \mathrm{r} p \mathrm{H}$ unit or I $\mathrm{mmHg}$ for $\mathrm{PCO}_{2}$. Mean readings were then rounded. Base excess or deficit has been calculated from $p \mathrm{H}, \mathrm{PCO}_{2}$, and haemoglobin, and approximated to the nearest whole number.

All arrhythmias considered in this study occurred within the 24 hours subsequent to arterial sampling, and later arrhythmias have not been included. Studied arrhythmias were: (I) ventricular tachycardia; and (2) ventricular premature beats, provided and only if they fulfilled at least one of the following criteria: (a) a frequency greater than 5 a minute, (b) multifocal in origin, (c) arose from a single focus but occurred in pairs or runs; and (d) exhibited the $R$ on $T$ phenomenon.

Sinus, and atrial arrhythmias, escape rhythms, heart block, and bundle-branch block have not been included. Ventricular fibrillation only occurred once, in the absence of cardiogenic shock, during the period of study. This fibrillation was pacemaker induced and has not been included.

The 142 episodes of myocardial infarction have been divided into three severity groups. The first group consists of those patients in whom there was no clinical or radiological sign of heart failure over the period of study. This group is considered uncomplicated. Should there have been such signs, patients were allocated to the second or heart failure group. The third group consists of patients with cardiogenic shock. Only four patients suffered this complication over the period of study and six others subsequently. All died.

The relation between acid-base status and the incidence of arrhythmias has then been assessed. This has been done both overall and within each severity group using unpaired t-tests or $\chi^{2}$ to determine statistical significance.

\section{Results}

These are displayed by histogram in Fig. $I$ and 2.

Overall results I04 patients $(87 \mathrm{men}$ and 17 women - mean age 56.0 ) did not sustain any ventricular arrhythmia over the period of study immediately after infarction. Their mean $p \mathrm{H}$ was 7.417 (standard deviation 0.05 ) and base-excess $+\mathrm{I} \cdot 0$ (SD 3:I). Arrhythmias were sustained by 38 patients ( $3 \mathrm{I}$ men and 7 women - mean age 58.0 ) - there being 24 patients with ventricular premature beats of the types mentioned, ro patients with ventricular tachycardia, and 4 shocked patients with agonal arrhythmias. The mean $p H$ of these 38 patients was 7.414 (SD 0.065$)$ and their base-excess +0.05 (SD 3.8). $t$ testing of these results indicates no difference in $p \mathrm{H}$ between the two groups $(\mathbf{P}>0 \cdot I)$ but gives an equivocal result for base-deficit/excess $(P<0.1>0.05)$. Accordingly the results have been broken down into severity groups in order to obtain a definite answer.

Uncomplicated patients 54 patients were free of complications and there were no deaths in this group. No patient had a metabolic acidosis (as defined by a base-deficit greater than -3), while ro patients had a metabolic alkalosis (as defined by a baseexcess greater than +3$) .46$ of these patients did not sustain arrhythmias; their mean $p H$ was 7.419 (SD 0.036) and base-excess $+\mathrm{I} \cdot 4$ (SD 2.4). Eight patients did undergo arrhythmias - all of whom were in normal acid-base balance; their mean $p H$ was 7.413 (SD 0.036 ) and base-excess $+r \cdot 0($ SD $r \cdot 8)$. $t$ testing indicates that $p \mathrm{H}$ and base-excess are essentially similar between the two groups $(P>0 \cdot I)$.

Patients with heart failure There were 84 patients with evidence of heart failure during the 24 hours after acid-base measurements and of whom 14 died during their hospital stay. Eight patients had a metabolic acidosis -2 of these sustaining arrhythmias while I6 had a metabolic alkalosis, arrhythmias occurring in 4 . The remaining 20 arrhythmic patients were in normal acid-base balance. Testing by $\chi^{2}$ suggests no relation between acidosis, alkalosis, and normal acidbase balance on the one hand and the presence or absence or arrhythmias on the other. This lack of relation is confirmed by $t$ testing -58 non-arrhythmic patients having a mean $p \mathrm{H}$ of 7.415 (SD 0.06) and base-excess of +0.7 (SD 3.5 ) while 26 arrhythmic patients had a mean $p \mathrm{H}$ of 7.429 (SD 0.055) and base-excess of $+0.9\left(\mathrm{SD}_{2} \cdot 7\right)$.

Patients with cardiogenic shock Four patients showed evidence of severe cardiogenic shock during the 24-hour period of study immediately after admission. Arterial samples were obtained from these patients (= early onset shock) and from 4 of the 6 patients who became shocked after the 24hour period of study (=late onset shock). All these patients died - thereby sustaining agonal arrhythmias - within 24 hours of sampling. Of these 8 patients, 5 had a metabolic acidosis ( 2 of them showing the greatest base deficit of the whole series) and none was alkalotic. Their mean $p H$ was 7.349 (SD 0.085 ) and base deficit - 5.I (SD 5.0). Since there was no shocked patient in whom arrhythmias did not develop, no direct comparisons can be made.

However, it does appear that cardiogenic shock per se has a significant association with acidosis $(p \mathrm{H} \quad \mathrm{P}<0.05$, base-deficit/excess 


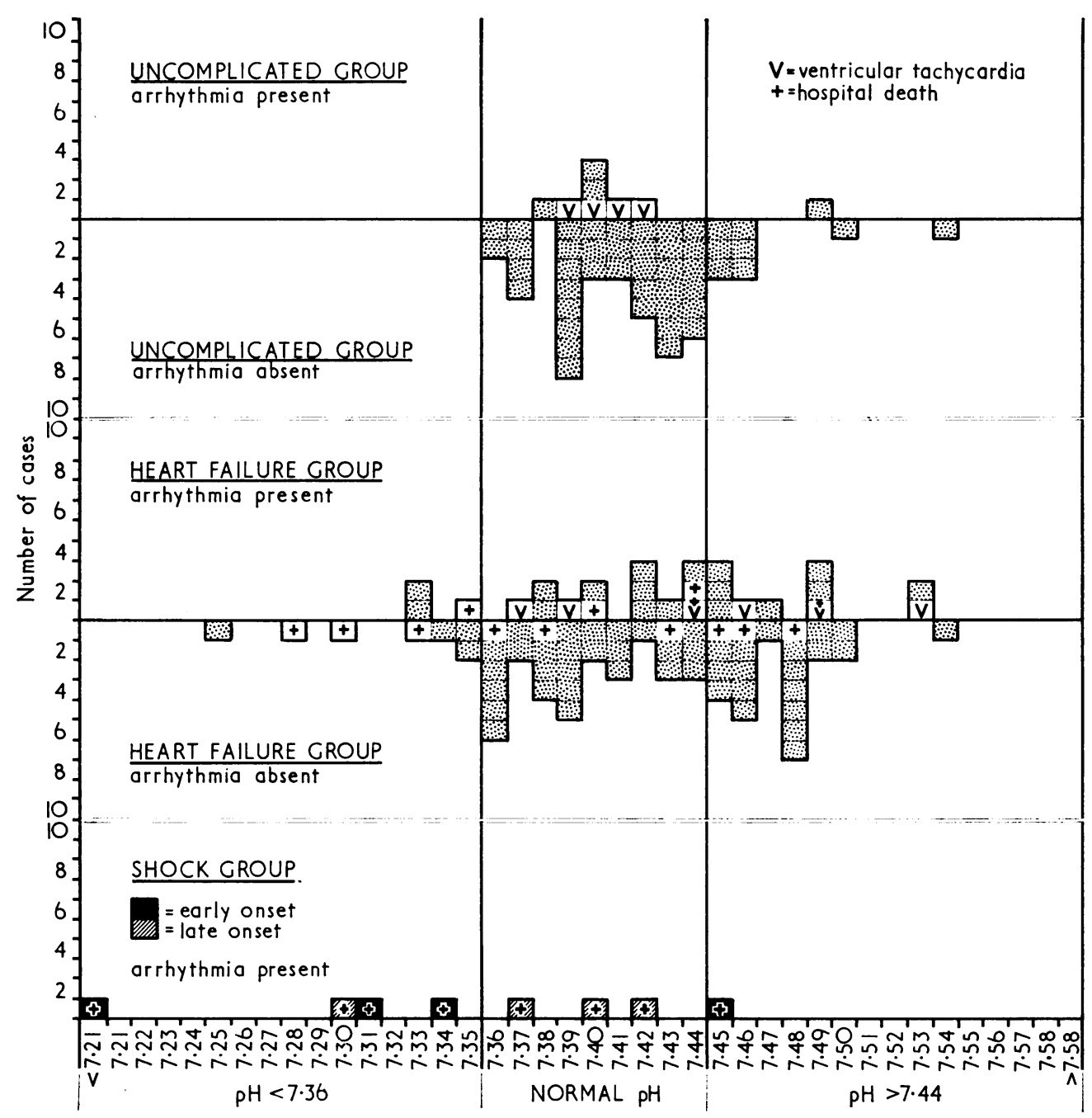

FIG I $p H$ and ventricular arrhythmias.

$\mathrm{P}<0.0 \mathrm{I}$ - as compared with acid-base status in non-shocked patients) and that shock carries an increased risk of arrhythmia ( $P<0.01$ on these figures). Evidently there is an association between acidosis, arrhythmias, and cardiogenic shock, but one cannot say whether the acidosis itself is the cause of such arrhythmias.

These findings suggest that the overall equivocal results obtained for comparison between base-deficit or excess and arrhythmias may be caused by the values obtained in cardiogenic shock. Indeed this is the case and if shock be excluded mean base-excess for 34 arrhythmic patients becomes +0.95 (SD 2.5) - clearly indicating that there is no relation between acid-base status and arrhythmias in myocardial infarction without cardiogenic shock.

\section{Relation between death and acid-base} balance Eighteen patients died; 4 of these succumbed to early onset cardiogenic shock and 6 to shock of late onset. The remainder all died after the 24-hour periods of study, death being caused by congestive cardiac failure in 4 patients, cardiac rupture, certainly in one and probably in another, and as a direct consequence of arrhythmia in the 2 remaining patients.

There is no association between alkalosis and death; indeed no patient with a metabolic alkalosis died. However, there is a very significant association between both low $p \mathrm{H}(<7 \cdot 36)$ 


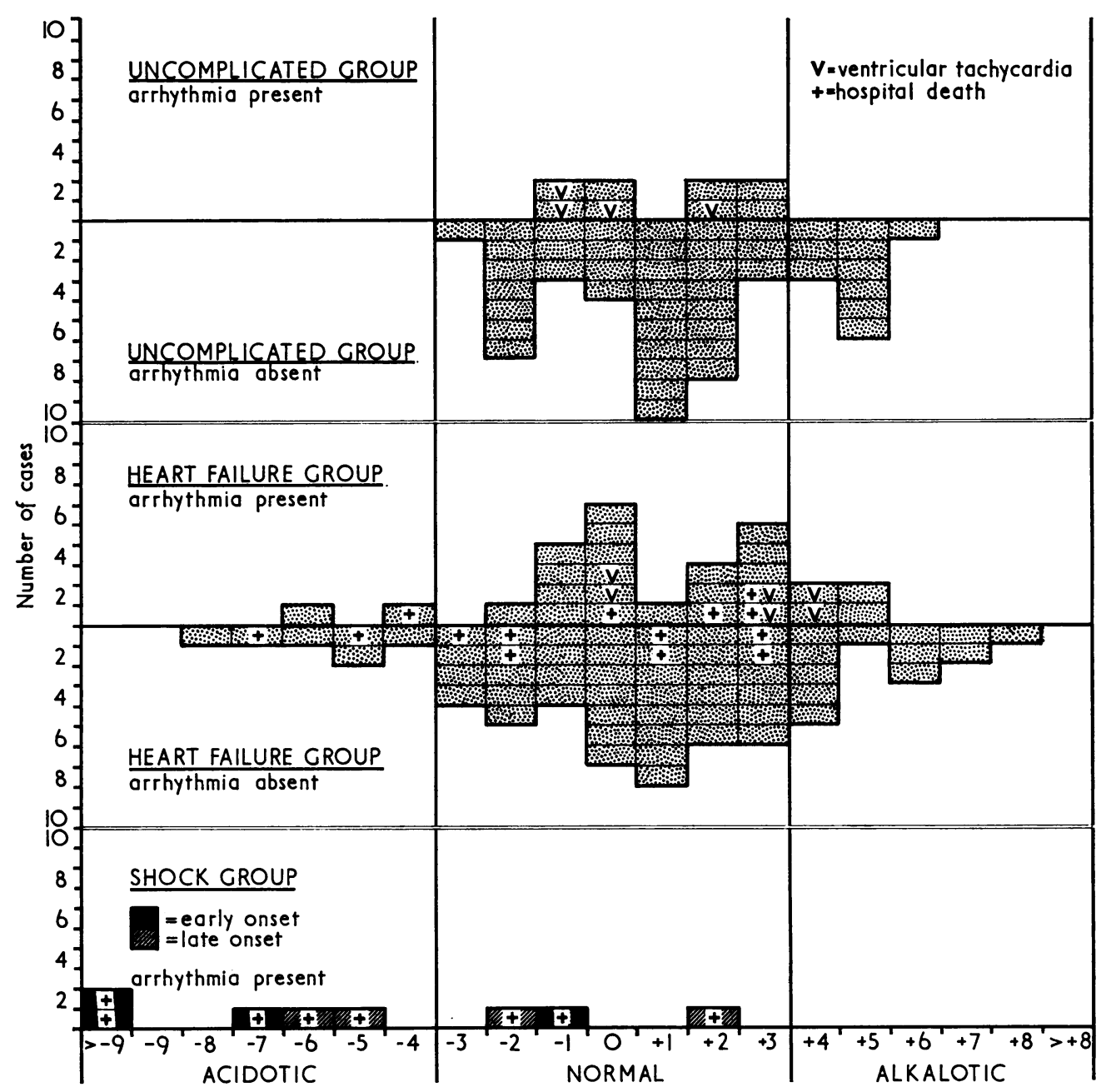

FIG 2 Base excess or deficit and ventricular arrhythmias

and metabolic acidosis and death $(P<0.001)$. Almost certainly this is the result of inadequate tissue perfusion via poor cardiac output.

\section{Discussion}

This study categorically shows that there is no relation between acid-base status and ventricular arrhythmias in myocardial infarction without cardiogenic shock. However, it stands or falls on two assumptions. Firstly, either all arrhythmias must have been correctly recognized and detected, or alternatively nonrecognition must have been random over the observed range of $p \mathrm{H}$ and base-deficit or excess. Secondly, either acid-base status remained constant over the period of measurement or alternatively movement in acid-base status over this time was random between the two groups of arrhythmic and non-arrhythmic patients.

This study indicates an incidence of 27 per cent ventricular arrhythmias over the time studied. This incidence is certainly much lower than the usually accepted incidences of between 73 per cent (Imperial, Carballo, and Zimmerman, 1960) and 95 per cent (Julian, Valentine, and Miller, 1964). These high figures do of course cover all types of arrhythmia observed after infarction and, also unlike this series, are not limited by time. Twentyeight patients in this study sustained potentially dangerous ventricular premature beats (being isolated in 24 cases and proceeding to ventricular tachycardia in 4) - an incidence of 20 per cent. It is difficult to state comparable figures, but the findings of Julian et al. (I964) and of Lown, Kosowsky, and Klein (I969) 
suggest an incidence of about 30 per cent for these types of premature beats over the course of infarction.

Comparisons with these figures certainly suggest that arrhythmias have been missed in this series. Indeed it would be surprising had they not been missed. However, nursing staff, on whom arrhythmia detection largely depended, though in general terms aware of the objects of the study were not informed about acid-base status in any individual patient, thus avoiding bias towards keener observations in acidotic or alkalotic patients. By this means it is felt that non-recognition problems do remain random.

$p \mathrm{H}$ is the sum of metabolic and respiratory acid-base status and rapidly affected by respiratory change. In a study such as this it is certainly affected by the often necessary prior analgesia and perhaps by arterial puncture itself despite careful technique. Fluctuations of $p \mathrm{H}$ over short periods of time must occur, and there is no guarantee or even likelihood that the value obtained represents their mean. Base-deficit or excess represents metabolic acid-base status only, and is free from rapid swings due to respiratory change. Nevertheless, a 24-hour period of observation after arterial puncture is a long enough time for almost certain changes in this measurement to occur - and particularly in an unstable state, such as after myocardial infarction. Short of on-line monitoring of $\mathrm{pH}$ and $\mathrm{PCO}_{2}$, there is no means of knowing whether movement in acid-base status was indeed random between the groups of arrhythmic and nonarrhythmic patients. It does seem empirically likely, but this assumption must remain unproven.

In general terms cardiac tachyarrhythmias are caused by an alteration in the firing rate of normally automatic specialized cells. Any factor that increases the automaticity of a group of cells outside the sinus node to a level above that of the sinus node will result in an arrhythmia. Many factors can do this and include acid-base imbalance. An increase in $\mathrm{PCO}_{2}$ causes an increase in the rate of automatic diastolic depolarization (Karis et al., 1960; Hoffman and Cranefield, 1964), while changes in $p \mathrm{H}$ not caused by changes in $\mathrm{PCO}_{2}$ may also reduce the maximum level of depolarization and thereby approximate it to the threshold firing value (Karis et al., 1960; Hecht and Hutter, 1964). At a more clinical level metabolic acidosis reduces the threshold for ventricular fibrillation (Gerst et al., 1966), and if it is not corrected after cardiac arrest, post-arrest arrhythmias are provoked (Ledingham and Norman, 1962).
Alkalosis probably exerts an effect on the firing rate of automatic cells by changing the distribution of ions between intracellular and extracellular fluids. Experimental respiratory alkalosis is known to shift both potassium and sodium into cells (Giebisch, Berger, and Pitts, 1955), and it is likely that metabolic alkalosis has a similar effect (Adler, Roy, and Relman, 1965). A decrease in extracellular potassium causes an increase in the rate of diastolic depolarization (Hoffman and Cranefield, 1964), and arrhythmias may ensue. Surprisingly, respiratory alkalosis was not found to alter the threshold for ventricular fibrillation in one study while metabolic alkalosis actually protected the heart from this arrhythmia (Gerst et al., 1966). However, the dangers of arrhythmias being provoked by mechanical hyperventilation are now well known (Flemma and Young, 1964), and recently two patients have been reported in whom recurrent ventricular fibrillation after myocardial infarction was associated with a respiratory alkalosis, and in whom the arrhythmia could not be corrected until ventilation was depressed (Ayres and Grace, 1969).

The production of arrhythmias after myocardial infarction is complex and certainly multifactorial. They are certainly related to the catecholamine release known to occur in myocardial infarction (Valori, Thomas, and Shillingford, 1967). The rate of diastolic depolarization is also increased by the passage of weak depolarizing currents across automatic cells (Trautwein and Kassebaum, 196I), and it has been suggested that the current of injury produced in dying or injured myocardium may enhance the automaticity of adjacent cells and thereby initiate arrhythmias (Hoffman, Cranefield, and Wallace, 1966). Localized myocardial ischaemia - such as is present in a peri-infarction zone - sensitizes these adjacent automatic cells to the influence of catecholamines (Hoffman and Cranefield, 1964). Generalized arterial hypoxaemia is common after myocardial infarction (Valencia and Burgess, 1969), and hypoxaemia has been shown to accelerate diastolic depolarization with possible arrhythmia production (Hoffman et al., 1966). Stretch is also known to have a similar effect (Hoffman et al., 1966), and may be a factor in the provocation of arrhythmias affecting those hearts dilated from heart failure.

The situation becomes more complex in view not only of the interrelationships of the various factors that may provoke arrhythmias but of their relation with cardiac contractility. Catecholamines certainly increase contractility but may do this at the expense of further 
myocardial anoxia (Yurchak et al., 1964), since the slight increase in coronary flow does not keep pace with augmented oxygen requirements. Acidosis is a cardiac depressant $(\mathrm{Ng}$, Levy, and Zieske, 1967) but a stimulant to catecholamine release in its own right (Malm et al., 1966). Catecholamine release protects the heart from acidotic depression (Rocamora and Downing, 1969), but below a certain $p \mathrm{H}$ level this protection probably fails. This protection failure was shown in dogs in whom the critical $p \mathrm{H}$ level was $7 \cdot \mathrm{I}$ (Wildenthal et al., 1968). In myocardial infarction acidosis and hypoxaemia are usually coexistent, and hypoxaemia has been shown to enhance greatly the myocardial depression of acidosis (Gelet, Altschuld, and Weissler, 1969).

At a clinical level, there seems likely to be a vicious circle mechanism set in action by severe infarction. With regard to cardiac output this is immediately reduced by the infarction. The ensuing poor tissue perfusion leads to metabolic acidosis. The heart is initially protected against this by the associated catecholamine release, but abetted by hypoxaemia this protection soon fails. The cardiac output then worsens - acidosis increases and cardiac output is yet further depressed, etc.

A somewhat similar situation can be envisaged with regard to arrhythmias. They may be provoked by the initial catecholamine release after infarction and probably are initiated from those ischaemic automatic cells adjacent to the infarct. Injury currents may be important at this site. More severe infarctions are likely to be complicated by the respiratory alkalosis and cardiac dilatation of left ventricular failure. Hypoxaemia may be produced by left ventricular failure or by other ventilation-perfusion abnormalities (Valencia and Burgess, 1969) and may be provoked at myocardial level by catecholamine release. Each of these factors may initiate arrhythmias in their own right or potentiate each other until arrhythmias are provoked. It seems likely that acid-base imbalance has little part to play in the initial production of arrhythmias. Metabolic acidosis may later play an important, but secondary role. Abetted by hypoxaemia it may become sufficiently severe to depress further cardiac output. This reduction in output will then aggravate most of those other factors concerned in arrhythmia production while the further acidosis produced by this fall in output will tend to provoke further arrhythmias, etc.

Whether acidosis can directly provoke arrhythmias in this situation is obscured by the other factors involved and by its probable secondary action in their production. It seems likely that acid-base imbalance must be gross before it plays a primary role. In experimental studies on isolated guinea-pig hearts very few arrhythmias were provoked over the $p \mathrm{H}$ range 6.95 to 7.48 (McElroy, Gerdes, and Brown, 1958), while in intact dogs there was no significant change in heart rate or rhythm over the $p \mathrm{H}$ range 7.23 to 7.39 (Goodyer et al., 1961). What little clinical evidence there is confirms this. The two patients described by Ayres and Grace (1969) with recurrent ventricular fibrillation due to respiratory alkalosis were both severely alkalotic - the $p \mathrm{H}$ in each being in excess of $7 \cdot 7$, while two postoperative patients described by Brooks and Feldman (1962) with alkali-responsive ventricular fibrillation were quite grossly acidotic, their base-deficits being in excess of -20 .

Despite the considerable volume of clinical and experimental evidence detailed above suggesting that acid-base imbalance does cause arrhythmias, the findings of this study are not in disagreement. To cause arrhythmias acid-base imbalance must be gross. Gross imbalance is not observed in myocardial infarction without prior cardiac arrest or cardiogenic shock. Consequently acid-base imbalance can play no more than the most trivial part in arrhythmia production under most circumstances. This study has shown an association between cardiogenic shock, acidosis, and arrhythmias. There is insufficient evidence from the study to determine whether there is any primary association between gross acidosis and arrhythmias or whether it be mediated via the inadequate cardiac output of shock. The evidence detailed above suggests that both factors are operative - the second probably being more important than the first - and that they are cumulative.

The results of this study confirm and amplify the conclusions of Anderson et al. (1968). They found that a metabolic acidosis of greater than -2.5 on admission was associated with an increased tendency to arrhythmias over the next three days. This acidosis was accompanied by the signs of an inadequate cardiac output (as shown by hypotension and cold extremities), and it was considered that the apparent association between acidosis and arrhythmias was probably related to the greater severity of infarction in these patients. Their findings are essentially similar to those of this study.

Little work has been done on the effects of alkali administration to patients with arrhythmias in myocardial infarction. Anderson et al. (1968) did give alkali to three acidotic hypotensive patients with arrhythmias. It did not correct these arrhythmias, though their 
general condition did improve. It certainly does seem logical to give alkali to acidotic patients and has been previously recommended by Neaverson (1966). Such alkali administration might be expected to act as a brake in the vicious circle mechanism of acidosis causing a further fall in cardiac output, etc., but would not be expected to exert much influence on arrhythmias whose genesis is multifactorial.

This study would not have been possible without the skill and enthusiasm of the nursing staff, Ward A4, Selly Oak Hospital, Birmingham. J. P. was in receipt of a Sheldon Research Fellowship from the Birmingham Regional Hospital Board. Retrospective statistical assistance was given by Mr. R. A. Dixon of the Department of Preventive Medicine, University of Sheffield.

\section{References}

Adler, S., Roy, A., and Relman, A. S. (1965). Intracellular acid-base regulation. I. The response of muscle cells to changes in $\mathrm{Co}_{2}$ tension or extracellular bicarbonate concentration. Fournal of Clinical Investigation, 44, 8.

Anderson, R., Gardner, F. V., Honey, H., Noble, I. M., and Woodgate, D. W. (I968). Relation between metabolic acidosis and cardiac dysrhythmias in acute myocardial infarction. British Heart fournal, 30, 493.

Ayres, S. M., and Grace, W. J. (1969). Inappropriate ventilation and hypoxemia as causes of cardiac arrhythmias. American fournal of Medicine, 46, 495.

Brooks, D. K., and Feldman, S. A. (1962). Metabolic acidosis, a new approach to neostigmine resistant curarisation. Anaesthesia, 17, 16r.

Flemma, R. J., and Young, W. G., Jr. (1964). The metabolic effects of mechanical ventilation and respiratory alkalosis in postoperative patients. Surgery, 56, 36.

Gelet, T. R., Altschuld, R. A., and Weissler, A. M. (1969). Effects of acidosis on the performance and metabolism of the anoxic heart. Circulation, 40, Suppl. 4, 60 .

Gerst, P. H., Fleming, W. H., and Malm, J. R. (1966). A quantitative evaluation of the effects of acidosis and alkalosis upon ventricular fibrillation threshold. Surgery, 59, ro5o.

Giebisch, G., Berger, L., and Pitts, R. F. (1955). The extrarenal response to acute acid-base disturbances of respiratory origin. fournal of Clinical Investigation, 34, 231.

Goodyer, A. V. N., Eckhardt, W. F., Ostberg, R. H., and Goodkind, M. J. (196I). Effects of metabolic acidosis and alkalosis on coronary blood flow and myocardial metabolism in the intact dog. American fournal of Physiology, 200, 628.

Hecht, H. H., and Hutter, O. F. (1964). Action of pH on cardiac Purkinje fibers. Federation Proceedings, 23, 157.

Hoffman, B. F., and Cranefield, P. F. (1964). The physiological basis of cardiac arrhythmias. American fournal of Medicine, 37, 670 .

Hoffman, B. F., Cranefield, P. F., and Wallace, A. G. (1966). Physiological basis of cardiac arrhythmias. Modern Concepts of Cardiovascular Disease, 35, 103.

Imperial, E. S., Carballo, R., and Zimmerman, H. A. (1960). Disturbance of rate, rhythm and conduction in acute myocardial infarction: a statistical study of 153 cases. American fournal of Cardiology, 5, 24.

Julian, D. G., Valentine, P. A., and Miller, G. G. (I964). Disturbance of rate, rhythm and conduction in acute myocardial infarction. A prospective study of 100 consecutive unselected patients with the aid of electrocardiographic monitoring. American Fournal of Medicine, 37, 915.

Karis, J. H., Harmel, M. H., and Hoffman, B. F. (I960). Effects of $\mathrm{pH}$ and $\mathrm{PCO}_{2}$ on the sensitivity of Purkinje fibers to digitalis. Circulation, 22, 770.

Ledingham, I. McA., and Norman, J. N. (1962). Acidbase studies in experimental circulatory arrest. Lancet, $2,967$.

Lown, B., Kosowsky, B. D., and Klein, M. D. (I969). Pathogenesis, prevention, and treatment of arrhythmias in myocardial infarction. Circulation, 40 , Suppl. 4, 26r.

McElroy, W. T., Jr., Gerdes, A. J., and Brown, E. B., Jr. (1958). Effects of $\mathrm{Co}_{2}$, bicarbonate and $\mathrm{pH}$ on the performance of isolated perfused guinea pif hearts. American fournal of Physiology, 195, 4I2.

MacKenzie, G. J., Taylor, S. H., Flenley, D. C., McDonald, A. H., Staunton, H. P., and Donald, K. W. (1964). Circulatory and respiratory studies in myocardial infarction and cardiogenic shock. Lancet, 2, 825.

Malm, J. R., Manger, W. M., Sullivan, S. F., Papper, E. M., and Nahas, G. G. (1966). The effect of acidosis on sympatho-adrenal stimulation; particular reference to cardiovascular bypass. fournal of the American Medical Association, 197, 121.

Neaverson, M. A. (1966). Metabolic acidosis in acute myocardial infarction. British Medical fournal, 2, 383.

$\mathrm{Ng}$, M. L., Levy, M. N., and Zieske, H. A. (1967). Effects of changes of $\mathrm{pH}$ and of carbon dioxide tension on left ventricular performance. American fournal of Physiology, 213, II5.

Rocamora, J. M., and Downing, S. E. (1969). Preservation of ventricular function by adrenergic influences during metabolic acidosis in the cat. Circulation Research, 24, 373.

Trautwein, W., and Kassebaum, D. G. (1961). On the mechanism of spontaneous impulse generation in the pacemaker of the heart. Fournal of General Physiology, 45, 317.

Valencia, A., and Burgess, J. H. (1969). Arterial hypoxemia following acute myocardial infarction. Circulation, 40, 641.

Valori, C., Thomas, M., and Shillingford, J. P. (1967). Urinary excretion of free noradrenaline and adrenaline following myocardial infarction. Lancet, I, 127.

Wildenthal, K., Mierzwiak, D. S., Myers, R. W., and Mitchell, J. H. (1968). Effects of acute lactic acidosis on left ventricular performance. American fournal of Physiology, 214, 1352.

Yurchak, P. M., Rolett, E. L., Cohen, L. S., and Gorlin, R. (1964). Effect of norepinephrine on the coronary circulation in man. Circulation, 30, 180. 